the long run include, myocardial infarction, stroke, renal failure, blindness, etc. Therefore, target values for biological markers have been determined below which organ damage does usually not occur and life expectancy is normalised. Examples for domains in which such thresholds have been defined are blood pressure, glycosylated haemoglobin $(\mathrm{HbA} 1 \mathrm{c})$, and others.

Inflammatory rheumatic diseases lead to organ damage not only in the musculoskeletal system but they may also harm internal organs. A target level of a measure related to its long-term outcome, can be a surrogate measure like the cholesterol level for cardiovascular diseases, or a composite measure of disease activity as used in RA (DAS28) or in AS (ASDAS). The treat-to-target strategy can be reduced to a simple algorithm of, on the one hand measuring activity and on the other hand, in consequence, adapting treatment. Treatment adaptation does not necessarily mean changing a medication or increasing the dosage of a drug but may even also mean life style changes, as long as the therapeutic target is attained or nearly attained - importantly within a prespecified time frame. Therapeutic adaptations should always take patient factors, including comorbidities, adverse events and patient preferences, into account

However, the musculoskeletal system can mostly not be assessed by using a simple surrogate or direct "gold standard" measures, since rheumatic diseasese with multiple signs and symptoms are mostly rather complex. In RA information derived from physical examination using a quantitative joint count is considered very important. This is different in AS. Additionally, information from the history, which can be collected through patient self-report multifaceted questionnaires, has proven effective in determining patient status and its change. This is even more important in AS. However, functional impairment has reversible and irreversible components. Damage is a consequence of high and/or persisting disease activity. The most important variables contributing to joint damage in RA are swollen joint counts and C-reactive protein (CRP). The latter in combination with questions on back pain is also important in AS, while in RA the use of composite measures of disease activity that comprise joint counts is critical. There is good evidence that, if this strategy is consequently followed, physical function will improve and joint damage be reduced in patients with RA. To determine optimal treatment targets in $\mathrm{RA}$ it is necessary to define the thresholds of disease activity measures at which progression of joint destruction is halted. Of note, only remission is associated with maximal reversal of functional impairment and a stop of progression of damage as well as work disability. However, it needs to be realized that some remission criteria are more stringent than others.

Disclosure of Interest: None declared

DOI: 10.1136/annrheumdis-2017-eular.7129

\section{SP0055 WHAT ARE THE CHALLENGES FOR APPLYING TREAT TO TARGET IN AXIAL SPONDYLOARTHRITIS?}

M. Dougados. rheumatology Hopital Cochin, René Descartes University, paris, France

The concept of Treat to Target (T2T) applied in rheumatoid arthritis has been evaluated in psoriatic arthritis and is currently under investigation in two different strategy trials in patients suffering from axial spondyloarthritis (axSpA). Whatever le results of these trials will be, the acceptation of this concept and consequently its implementation in daily practice might be challenging for several reasons. The concept of T2T necessitates 3 different steps and also the close collaboration of the patient and the availability of different treatment modalities.

The three different steps consist in:

a) the choice of the most relevant outcome measure (e.g. a measure evaluating a domain recognized as predisposing to subsequent clinically relevant damage (either structural damage or important comorbidities such as cardiovascular diseases)).

b) the determination of the threshold of the outcome measure to reach (threshold below which the risk of subsequent damage is abolished or significantly decreased).

c) The time to reach the target is usually related to the treatment modality (a few days for NSAIDs and several weeks for DMARDs).

A part these different steps, two points have to be considered a) this T2T approach is impossible without embarking the patient in a true share decision b) this T2T strategy requires the possibility to adapt/increase the treatment in case the target is not reached after one or several "conventional" treatment modalities.

For each of these different points we will consider past-on ongoing initiatives proposing to resolve the different encountered issues in order to facilitate the elaboration and the implementation of a T2T strategy in AxSpA.

Disclosure of Interest: $M$. Dougados Grant/research support from: UCB,ABBVIE,PFIZER,MERCK, Consultant for: UCB,ABBVIE,PFIZER,MERCK

DOI: 10.1136/annrheumdis-2017-eular.7119

\section{SP0056 UPDATE OF THE T2T RECOMMENDATIONS IN SPA}

D. Van Der Heijde on behalf of T2T in SpA working group. Rheumatology, Leiden University Medical Center, Leiden, Netherlands

In 2013 the first recommendations for treating spondyloarthritis to target (T2T) were published. These followed the reasoning for the T2T recommendations for rheumatoid arthritis. Although the systematic literature review at that time did not provide evidence to support the recommendations, five overarching principles and 11 recommendations were formulated. There were 9 commen recommendations for axial $\mathrm{SpA}$, peripheral $\mathrm{SpA}$ and psoriatic arthritis and 2 additional recommendations for each subgroup specifically. In 2017 the T2T working group met again to update the recommendations. This was based on an updated systematic literature review. Data had been published that there is indeed a clear link between inflammation and subsequent longterm outcomes, which is the basis for the T2T principles.

$\mathrm{SpA}$ is characterised by musculoskeletal signs and symptoms (arthritis, enthesitis, dactylitis, axial disease) but also extra-articular manifestations (psoriasis, inflammatory bowel disease, anterior uveitis) are important manifestations. Moreover, comborbidities (such as osteoporosis, cardiovascular disease). All these manifestations are taken into account in the formulation of the recommendations. The overarching principles were kept largely identical. Some changes in the wording were made for a better understanding, but no fundamental changes were made. A total of 11 recommendations were formulated. These are now for all subgroups of SpA and no specific recommendations are proposed. In principle, the treatment target is remission or inactive disease of musculoskeletal and extra-articulaur manifestations, and the target should be individualised. It is important that remission/inactive disease should be based on a combination of clinical and laboratory parameters, and disease activity should be measured on the basis of clinical signs and symptoms as well as acute phase reactants. This is important to realise, e.g. in axial SpA as patient reported outcomes only are at best weakly correlated with structural damage. In certain circumstances, low disease activity may be an alternative target. Because of the heterogeneous presentation of SpA, not only the target, but also the assessments should be individualised. Both in the overarching principles and in the recommendation the shared decision between patient and rheumatologist is listed as the basis of the T2T management.

The updated recommendations will be presented.

Disclosure of Interest: D. Van Der Heijde Consultant for: AbbVie, Amgen, Astellas, AstraZeneca, BMS, Boehringer Ingelheim, Celgene, Daiichi, Eli-Lilly, Galapagos, Gilead, Janssen, Merck, Novartis, Pfizer, Regeneron, Roche, Sanofi, UCB, Employee of: Imaging Rheumatology DOI: 10.1136/annrheumdis-2017-eular.7158

\section{THURSDAY, 15 JUNE 2017 Calcium crystal deposition in rheumatic diseases _}

\section{SP0057 CALCIUM CRYSTALS AND THEIR LINK TO OSTEOARTHRITIS}

\section{J. Bertrand. Department of Orthopeadic Surgery, Otto-von-Guericke University} Magdeburg, Magdeburg, Germany

Calcification of cartilage is a common finding during osteoarthritis (OA). We have shown that it is mainly of the BCP type and not the CPPD type of crystal formation. BCP cartilage calcification is directly linked to the severity of cartilage degradation and, therefore, OA severity. We have also shown that with increasing hypertrophic differentiation of chondrocytes, the amount of calcification increases in vivo and in vitro. This indicates a link between chondrocyte hypertrophy andcartilage calcification. The pyrophosphate pathway is known to be involved in tissue calcification. It functions to keep the sensitive balance of pyrophosphate (PPi) and phosphate $(\mathrm{Pi})$, thereby preventing the generation of calcium-phosphate crystals. One key player in this pathway is the nucleotide pyrophosphatase phosphodiesterase (NPP1), which has been demonstrated to be regulated by inflammatory mediators such as IL-1. In our cohort of OA patients, the expression of collagen X and NPP1, but not ANK and TNAP, correlated with cartilage calcification and also with the Mankin-Score. NPP1 expression inverse correlated with the calcification, whereas collagen $X$ was upregulated. This finding was confirmed in experimental murine OA using the DMM mouse model. Furthermore, NPP1mut/mut mice (ttw/ttw) exhibit more calcification activity than wild type controls in joints as well as in cartilage of non weight bearing areas, including ear cartilage, suggesting that mechanical stress is not required for the induction of calcification. NPP1mut/mut (ttw/ttw) mice developed typical OA-like changes as evaluated by histological analysis as well as in vivo imaging and histological stainings. Intriguingly, calcification was associated with increased expression of the hypertrophic cartilage marker collagen $\mathrm{X}$ and the bone marker collagen I. Additionally, BCP crystals are able to activate chondrocyte differentiation via the WNT signaling pathway.

NPP1 is an important player in OA-associated cartilage calcification. Pathologic calcification of cartilage resembles in many aspects cartilage transformation into bone. Taken together, the data suggest that $\mathrm{OA}$ is characterized by the re-activation of molecular signalling cascades that at least in part resemble endochondral ossification.

Disclosure of Interest: None declared

DOI: 10.1136/annrheumdis-2017-eular.7192

\section{SP0058 REVIEW OF THE DIFFERENT IMAGING MODALITIES TO DETECT CALCIUM DEPOSITION DISEASES}

P. Omoumi. Lausanne University Hospital, Lausanne, Switzerland

Crystal deposits in and around the joints are common and most often encountered 